\title{
Kommentare
}

\section{Katja Kruse/Sabine Sczesny \\ Vergewaltigung und sexuelle Nötigung - bagatellisierende Auslegung und Scheitern einer Reform}

\section{Die gegenwärtige Strafverfolgungspraxis - Widersprüche und Ungleichzeitigkeiten}

Immer wieder lesen wir in der Zeitung von Strafverfahren wegen Vergewaltigung, die mit einer harten Strafe, etwa zwischen drei und fünf Jahren, selten sogar mehr, enden. Umgekehrt wird von unverständlichen Einstellungen oder Freisprüchen berichtet. Welche rechtlichen Reaktionsformen stehen hinter diesen widersprüchlichen Meldungen?

Beginnen wir mit den offiziell registrierten Zahlen. 1990 wurden etwa 5 100 Vergewaltigungen von der Polizei registriert. Die von betroffenen Frauen angezeigten Vergewaltigungen sind rückläufig. I 960 waren es etwa 6400 Fälle, 1980 stiegen sie auf knapp 7000. Zugleich wurde in dieser Zeit die Strafverfolgung - gemessen an früher und auch gemessen an den heute üblichen Standards - intensiver':

Tabelle I: Strafanzeigen wegen Vergewaltigung $1981-1990$

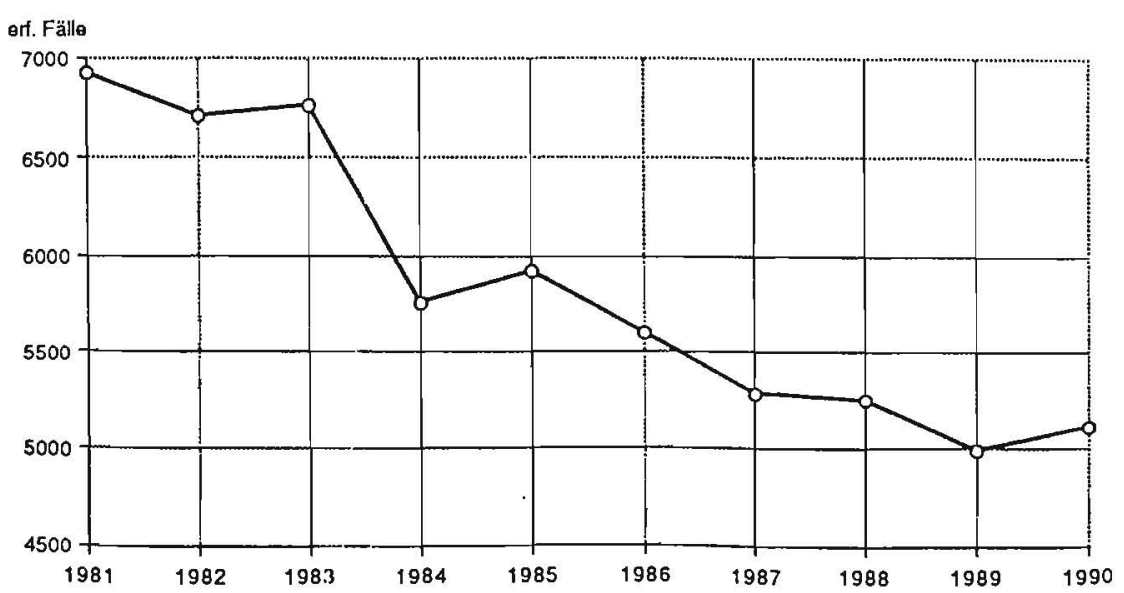

Quelle: Polizeiliche Kriminalstatistik

\footnotetext{
I Die folgenden Tabellen sind die aktualisierte Version aus: Monika Frommel, Systematische Verzerrungen bei der Umsetzung gesetzlicher Strafdrohungen - dargestellt am Beispiel der bereichsspezifischen Gewaltbegriffe der Strafverfolgungsinstanzen -, in: Raiser/Voigt (Hrsg.), Durchsetzung und Wirkung von Rechtsentscheidungen, Baden-Baden 1990, S. I 2 Iff., Tabelle 3 und 4, S. I 39; vgl. dies., ZfSexf I989, S. I $32 \mathrm{ff}$.
} 
Tabelle 2: Entwicklung der Verurteiltenziffern seit 1900 pro 100000 der Bevölkerung

\begin{tabular}{lrrrrrrrrr}
\hline Delikt & 1900 & 1910 & 1920 & 1930 & 1950 & 1960 & 1970 & 1980 & 1990 \\
Vergewaltigung & 2,5 & 1,8 & 1,0 & 2,0 & 1,9 & 4,5 & 3,8 & 2,3 & 1,7 \\
Vorsätzliche & & & & & & & & & \\
Körperverletzung & 306,0 & 258,0 & 105,0 & 100,0 & 40,0 & 60,0 & 47,0 & 57,1 & 54,0 \\
Raub & 1,1 & 1,5 & 3,2 & 2,1 & 3,1 & 3,7 & 5,9 & 6,0 & 6,1 \\
\hline
\end{tabular}

Quelle: Statistisches Jahrbuch 1992, S. 401

Tabelle 3: Nach allgemeinem Strafrecht Verurteilte nach der Dauer der Freiheitsstrafe

\begin{tabular}{|c|c|c|c|c|c|c|c|c|}
\hline \multirow[t]{2}{*}{ Delikte 1990} & \multirow[t]{2}{*}{$\begin{array}{l}\text { Ver- } \\
\text { urteilte } \\
\text { ins- } \\
\text { gesamt } \\
\\
1\end{array}$} & \multirow[t]{2}{*}{$\begin{array}{l}\text { Zu Freiheits- } \\
\text { strafe Ver- } \\
\text { urtelte } \\
\text { insgesamt } \\
2\end{array}$} & \multicolumn{2}{|c|}{$\begin{array}{l}\text { Regelmaßig aus- } \\
\text { setzungsfahige } \\
\text { Freiheitsstrafe } \\
\text { bis zu einem } \\
\text { Jahr }\end{array}$} & \multicolumn{2}{|c|}{$\begin{array}{l}\text { Unter besonde- } \\
\text { ren Umstanden } \\
\text { aussetzungs- } \\
\text { fahige Freiheits- } \\
\text { strafen von mehr } \\
\text { als einem Jahr } \\
\text { bis einschl. zwei } \\
\text { Jahren }\end{array}$} & \multicolumn{2}{|c|}{$\begin{array}{l}\text { Nicht mehr aus- } \\
\text { setzungsfahige } \\
\text { Freiheitsstrafen }\end{array}$} \\
\hline & & & $\mathrm{N}$ & $\begin{array}{l}\% \text { von } \\
2\end{array}$ & $\mathrm{~N}$ & $\begin{array}{l}\% \text { yon } \\
2\end{array}$ & $\mathrm{~N}$ & $\begin{array}{l}\% \text { von } \\
2\end{array}$ \\
\hline $\int \$ 177,178$ & 1230 & I 192 & 313 & 26,3 & 233 & 19,5 & 480 & $4^{\circ}, 3$ \\
\hline$\$ 177$ & 771 & 762 & I3I & 17,2 & 144 & 18,9 & 399 & 52,4 \\
\hline$\$ 178$ & 459 & 430 & I 82 & 42,3 & 89 & 20,7 & $8 \mathrm{I}$ & 18,8 \\
\hline $\mathbb{S} \$ 249,250,25 \mathrm{I}$ & 3131 & 2945 & 718 & 24,4 & 464 & Is, 8 & 1172 & 39,8 \\
\hline$\$ 240,234,24 \mathrm{Ia}$ & 6833 & 744 & 453 & 60,9 & 32 & 4,3 & 66 & 8,9 \\
\hline $\begin{array}{l}\text { Verbrechen } \\
\text { und Vergehen } \\
\text { insgesamt }\end{array}$ & 373007 & 79863 & 46905 & 58,7 & 5796 & 7,3 & 5788 & 7,2 \\
\hline
\end{tabular}

Quelle: Rechtspflegestatistik, Reihe 3, Strafverfolgung (eigene Berechnungen)

Tabelle 4: Freispruch und Einstellung des Verfahrens durch das Gericht bei den nach allgemeinem Strafrecht Abgeurteilten

\begin{tabular}{lccccc}
\hline Delikt & Jahr & $\begin{array}{l}\text { Abgeurteilte } \\
\text { N=100\% }\end{array}$ & $\begin{array}{l}\text { Einstellung } \\
\text { (Gericht) } \\
\%\end{array}$ & $\begin{array}{l}\text { Freispruch } \\
\%\end{array}$ & $\begin{array}{l}\text { nicht voll } \\
\text { Verurteilte } \\
\text { insg. \% }\end{array}$ \\
\hline$\$ 177$ & 1968 & 907 & $\mathrm{I}, 8$ & 31,2 & 34,3 \\
& 1980 & 1251 & 6,0 & 22,3 & 29,0 \\
& 1990 & 771 & 8,1 & 22,4 & 32,0 \\
\hline$\$ \$ 249-255,316$ & 1968 & 1934 & $4, \mathrm{I}$ & 18,2 & 22,9 \\
& 1980 & 3270 & 12,8 & 15,3 & 28,5 \\
& 1990 & 3131 & 18,0 & 14,2 & 33,4 \\
\hline alle Delikte ohne & 1968 & 319627 & 8,1 & 8,3 & 16,5 \\
Straßenverkehr & 1980 & 320294 & 17,9 & 6,6 & 24,6 \\
& 1990 & 373007 & 22,8 & 5,1 & 28,4 \\
\hline
\end{tabular}

Quelle: Rechtspflegestatistik, Reihe 3, Strafverfolgungen (eigene Berechnungen) 
Seit 1984 gehen die Anzeigen wegen Vergewaltigung deutlich zurück. Theoretisch ist es zwar denkbar, daß sexuelle Gewalt gegen Frauen tatsächlich seltener wird. Sehr viel plausibler ist aber die Annahme, daß es sich mittlerweile herumgesprochen hat, wie belastend gerichtliche Verfahren für die betroffenen Frauen sind. War früher die Behandlung durch die Polizei besonders problematisch, sind es mittlerweile die Gerichte $^{2}$. Offenbar haben sich zwischen den Strafverfolgungsbehörden die Kontrollstile gegenüber Beschuldigten und Zeugin bzw. Nebenklägerin strukturell verändert. Es ist mittlerweile die höchstrichterliche Rechtsprechung, die in erster Linie Anlaß zu Kritik gibt.

Aus Tabelle 4 ergibt sich, daß mehr als die Hälfte aller angezeigten Vergewaltigungen eingestellt oder wegen geringfügiger Vergehen verfolgt werden. Dies erklärt die niedrige Zahl der Abgeurteilten, also der Personen, die wegen Vergewaltigung vor Gericht angeklagt wurden. Die Selektivität der Strafverfolgung läßt sich anhand vorliegender Zahlen illustrieren: Von den genannten 5 s 00 angezeigten Vergewaltigungen werden etwa I 200 gerichtlich abgeurteilt. Darunter sind 994 Erwachsene, von denen wiederum 766 verurteilt werden. Berechnet man die Quote der Verfahren, in denen der Beschuldigte nicht voll verurteilt wird, dann beträgt diese 1990 etwa ein Drittel. Dies bedeutet aber zugleich, daß die Intensität der Strafverfolgung, die in den $1980 e r$ Jahren gestiegen war, in den i $990 e r$ Jahren wieder schwächer wird. Etwa die Hälfte aller verurteilten Täter werden nach dem unbenannten Strafmilderungsgrund des $\$ 177$ Abs. 2 StGB (sechs Monate bis zu fünf Jahren) wegen einer Vergewaltigung in einem minderschweren Fall relativ milde bestraft. Ein Viertel der Verurteilten muß mit Strafen über drei Jahren rechnen. Die Strafverfolgungs- und Strafzumessungspraxis ist also geprägt durch hohe Selektivität und eine dadurch verursachte Polarisierung der Delikte. Neben einer häufig zu beobachtenden, von der höchstrichterlichen Rechtsprechung nahe gelegten bagatellisierenden Auslegung kommt es zu Verfahren, in denen der Täter zum "Sündenbock « einer relativ weit verbreiteten sexuellen Gewalt gegen Frauen gemacht wird.

Die rechtlichen Reaktionsformen folgen einem widersprüchlichen Muster. Manche Täter werden sehr hart bestraft, in anderen Verfahren kommt es zu Einstellungen oder Freisprüchen bzw. Umdefinitionen des zugrunde liegenden Ausgangskonfliktes, die rational kaum nachvollziehbar sind. Im übrigen sind die Muster der Selektivität und Polarisierung regional unterschiedlich. Neuere Studien ergeben, daß insbesondere dort, wo staatsanwaltliche Sonderdezernate aktiv sind, die Strafverfolgung und Strafzumessung gleichmäßiger (dies bedeutet z. Zt. intensiver) ist. ${ }^{3}$

Frauenpolitische Aktivitäten sehen sich also vor der Schwierigkeit, für eine insgesamt konsequentere und damit im Ergebnis wohl härtere Strafverfolgung bei Vergewaltigung einzutreten, ansonsten aber vor einer Überschätzung des Strafrechts im allgemeinen zu warnen.

Das Thema »sexuelle Gewalt gegen Frauen« führt im strafrechtlichen Diskurs ein Schattendasein, ganz im Gegensatz zur allgemeinen politischen Diskussion. Lediglich eine kleine Reihe von - wegen ihrer Unverfrorenheit schlechthin nicht zu ignorierenden - Urteilen wurde rezipiert ${ }^{4}$. Hinterfragt wurden zwar der Gewaltbe-

2 Wiebke Steffen, Famılienstreıtigkeiten und Polızei, Kriminalıstik I2/1992, S. $800 \mathrm{ff}$

3 Claudia Kroll, Vergewaltigungsprozesse. Die gegenwartige Situation der Opfer von sexueller Gewalt im Gerichtsverfahren und Móglichkeiten zur Verbesserung (Kieler Notruf und Beratung fur vergewaltigte Frauen und Madchen e. V.), Kiel I 992.

4 BGH NJW 1981, S. 2204 ff. ("Lehrherr-Fall «); BGH NJW 1989, S. 3028 ("Hanauer Arzt-Fall «); vgl. zur kargen Rezeption Schapira, KJ x977, S. 22 Iff.; Goy, KJ 1987, S. 313 ; Wolter, NStZ 1985, S. 193 und S. 245 ; Sick, Sexuelles Selbstbestimmungsrecht und Vergewaltıgungsbegriff, 1993; Frommel, in: Bollınger/Lautmann (Hrsg.), FS fur Herbert Jager, 1993, S. 1 i 4 ff. 
griff und die Straflosigkeit der Vergewaltigung in der Ehe, nicht jedoch Struktur und Auslegung der Tatbestände der $\$ \$ 177$ und 178 StGB. Eine Reformdiskussion findet ausschließlich in einem kleinen Teil der Fachöffentlichkeit statt. Diese Ignoranz begünstigte eine Entwicklung in der Rechtsprechung, die durch eine Kombination unterschiedlicher Auslegungsschritte den Anwendungsbereich der sexuellen Gewaltdelikte erheblich einschränkt ${ }^{5}$. Die Basis für diese Einschränkung bilden die in diesem Bereich des Strafrechts nach wie vor fest verankerten patriarchalischen Strukturen. Untersucht werden im folgenden die Anforderungen, die an das Vorliegen einer Vergewaltigung i.S. d. $\$$ I 77 StGB oder einer sexuellen Nötigung i.S. d. $\$ 178$ StGB gestellt werden. Es sollen die Probleme der Tatbestände und daran anknüpfende Reformansätze dargelegt werden.

\section{Probleme der $\int S 177$ und 178 StGB in der Rechtsprechung}

Im Vergleich zu anderen Delikten sind im Bereich der Vergewaltigung und der sexuellen Nötigung immer noch signifikant höhere Einstellungs- und Freispruchsquoten festzustellen ${ }^{6}$. Sexuelle Gewalt wird bagatellisiert, das Verhalten der verletzten Frau gerät in den Mittelpunkt des Verfahrens. Es kommt zu einer sekundären Viktimisierung.

\section{Der Gewaltbegriff}

Der Begriff „Gewalt « begegnet uns heute nicht nur im Falle von Kriegen, Folterungen und Mord, sondern auch in den Bereichen Sport, Schule, Familie und Erziehung und als Gewalt gegen Ausländer. Zum einen hat der Begriff selbst im Laufe der Geschichte einen semantischen und funktionalen Wandel durchlaufen, zum anderen gibt es Differenzen in seiner Auslegung im Alltag7 und im Recht, in Recht wiederum in unterschiedlichen Verwendungen bei verschiedenen Straftatbeständen. Bestimmte Definitionen von Gewalt entsprechen dabei bestimmten Interessen, im Recht wie im Alltag $^{8}$. Je nach Bedarf werden dabei Strategien der Begriffserweiterung oder-verengung genutzt. Besonders deutlich wird dies durch die höchst unterschiedliche Auslegung des strafrechtlichen Gewaltbegriffes in den $\$ \$ 177,178$ StGB einerseits und $\$ 240$ StGB andererseits.

Sowohl $₫ 177$ I StGB wie $₫ 178$ I StGB fordern eine Nötigung »mit Gewalt« oder "durch Drohung mit gegenwärtiger Gefahr für Leib oder Leben«. Da das StGB keine Legaldefinition des Begriffs "Gewalt « enthält, muß sein Inhalt durch Auslegung bestimmt werden. Eine Nötigung "mit Gewalt « findet sich ebenso in $\$ \$ 240 \mathrm{I}$, 249 I und 253 I StGB. Es wäre daher naheliegend, allen Vorschriften einen ähnlichen Gewaltbegriff zugrundezulegen. Festzustellen ist dagegen eine unterschiedliche deliktsspezifische Auslegung.

Die Entwicklung des Gewaltbegriffes in $\$ 240$ I StGB ist gekennzeichnet durch eine zunehmende Ausweitung zu einem Sammelbecken für alle Zwangsformen außerhalb der Drohung, in deren Verlauf die Kriterien der Aufwendung körperlicher Kraft beim Täter oder einer körperlichen $\mathrm{Zwangswirkung} \mathrm{beim} \mathrm{Opfer} \mathrm{immer} \mathrm{mehr} \mathrm{zu-}$

\footnotetext{
s Vgl. Frommel, in: Bollinger/Lautmann (Hrsg.), FS fur Herbert Jager, 1993, S. 1 I 4 ff. (1 19).

6 Vgl. Abel, Vergewaltigung - Stereotypen in der Rechtsprechung und empirische Befunde, I986, S. 47; Rieß, 1n: FS fur Herbert Trondle, 1989, S. 369 ff. (374) wenn auch die Verurtellungsquote seit 1977 leicht ansteigend ist: Abel, S. 59; Rieß, S. 374.

7 Vgl. Schneider, Monatsschrift fur Kriminologie, 1990, S. 399 ff.

8 Neidhart, in: BKA (Hrsg.), BKA-Forschungsreihe: Sonderband "Was ist Gewalt? « Bd. 1, 1986, S. [13.
} 
rücktraten9. Ihren Höhepunkt fand diese »Vergeistigung « des Gewaltbegriffes im Laepple-Urteil, in dem der BGH sowohl auf das Erfordernis eines körperlichen Kraftaufwandes des Täters als auch auf die physische Einwirkung auf den Körper des Opfers verzichtet und eine lediglich psychische Einwirkung genügen läßt ${ }^{10}$. In der Rechtsprechung des BGH seither wird am Gewaltbegriff des Laepple-Urteils der Sache nach festgehalten, wenn auch nicht mehr ausdrücklich auf eine lediglich "psychische Einwirkung « Bezug genommen, sondern vielmehr ein »nicht nur seelischer, sondern auch körperlicher Z wang " beim Opfer gefordert wird" . Jedoch sind die an den »körperlichen Zwang « gestellten Anforderungen äußerst gering. So wurde beispielsweise in der Einwirkung durch "Geräuschentwicklung (Gebrüll, Pfeifen u. a.) auf einen Dozenten Gewaltausübung i.S. d. $\$ 240 \mathrm{StGB}_{\text {gesehen }}{ }^{12}$.

Festzustellen ist somit, daß der Sache nach weiterhin psychisch vermittelte $Z$ wangswirkungen beim Opfer vom Gewaltbegriff des $\$ 240$ StGB erfaßt werden, der Umfang der von der Rechtsprechung hier als ausreichend anerkannten Gewalt ist also sehr weit.

Der Inhalt des Gewaltbegriffes in $\mathbb{S} \mathbf{1} 77, \mathbf{1} 78 \mathrm{StGB}$ ist in beiden Vorschriften identisch zu bestimmen ${ }^{13}$, über die Anforderungen, die an das Vorliegen von Gewalt zu stellen sind, besteht in Literatur und Rechtsprechung jedoch keine Einigkeit. Vorherrschend ist ein restriktiveres Verständnis des Gewaltbegriffes als in $\$ 240 \mathrm{StGB}^{14}$. Maßgeblich soll hier die Position der Rechtsprechung sein, da sie sich unmittelbar auf das Opfer auswirkt.

Danach genügen »zur Gewaltanwendung alle eine gewisse - nicht notwendig erhebliche - körperliche Zwangseinwirkung darstellenden Handlungen, die von der Person, gegen die sie gerichtet sind, als ein nicht nur seelischer, sondern auch körperlicher Zwang empfunden werden ${ }^{15}$. Zum Teil wird auch zugestanden, für einen »körperlichen $Z$ wang « reiche es aus, »daß ein psychisch determinierter Prozeß mit nur geringem körperlichen Kraftaufwand in Lauf gesetzt wird ${ }^{16}$.

Hier wird vordergründig formuliert wie bei $\$ 240 \mathrm{StGB}$, doch sind die tatsächlichen Anforderungen an das Vorliegen von Gewalt erheblich höher. Letztlich entscheidend ist eine körperliche Kraftentfaltung beim Täter oder eine körperliche Zwangswirkung beim Opfer, eine psychisch vermittelte $Z$ wangswirkung allein wird nicht anerkannt. Dies wird ausdrücklich klargestellt. Das Merkmal der Gewalt sei »auch in den Fällen psychischer Zwangswirkung nur dann gegeben «, "wenn sie durch physische Kraftentfaltung vermittelt ist ${ }^{17}$. Der BGH betont, der Begriff der Gewalt könne nicht völlig vom Erfordernis körperlicher Kraftaufwendung gelöst wer$\operatorname{den}^{18}$.

Nicht anerkannt werden damit verbale Einwirkungen, selbst wenn sie mit einem psychischen Zwang von einigem Gewicht verbunden sind ${ }^{19}$. Ebenso soll ein "Spreizen der Beine« »ohne nennenswerten Kraftaufwand« nach vorangegangener zwei-

9 Vgl. zur Entwicklung des Gewaltbegriffes: Krey, in: BKA (Fn. 8), S. 28 ff.

10 BGHSt 23, S. 46 ff. ( 54 ).

I BGH NStZ 1982, S. I $88 \mathrm{ff}$. (I 59); BGHSt 37, S. 3 50 ff. (353)

12 BGH NStZ ig82, S. I $58 \mathrm{ff}$. (I 59 ).

${ }_{13}$ Krey (Fn. 8), Rn. I4I.

I4 Krey (Fn. 8), Rn. 149 m. w. N.; LK-Laufhutte, Io. Auf1. $1988 \$ 177$ Rn. 4, 6; Schonke/Schroder-Lenckner, 24. Aufl. 1991, $\$ 177$ Rn. 4 .

is BGHR StGB $\$ 177$, Gewalt 3, S. 1; BGH NStZ 1981, S. 218 ; BGH NStZ 1985, S. 71

I 6 BGHR StGB $\$ 177$, Gewalt 3, S. 1; BGH NStZ 1985, S. 7 1.

17 BGH NStZ 1981, S. 2 i 8.

18 BGH NStZ 1981, S. 218.

19 BGH NStZ 1981, S. 218 . 
maliger Vergewaltigung des Opfers durch andere Täter nicht zur Annahme von

Gewalt i.S.v. $\ 177 \mathrm{StGB}$ ausreichen ${ }^{20}$.

Besonders problematisch sind die Fälle, in denen die Frau an einen abgelegenen Ort gefahren oder in einem Raum eingeschlossen wird. Diese Handlungen sind zum Teil als Gewaltanwendung i.S. d. \I 77 StGB anerkannt worden ${ }^{21}$. Im Urteil zum »Lehrherr-Fall «22 stellte der BGH jedoch klar, daß keineswegs »in der einen oder anderen Art eines solchen Vorgehens « oder »in der Kombination beider « eine »tatbestandsmäßige Nötigung mit Gewalt zu sehen ist«. Vielmehr sei »in jedem Einzelfall auf Grund des gesamten Verhaltens des Täters und der durch ihn für die Frau geschaffenen Lage zu prüfen, ob die in $\$ 177$ StGB vorausgesetzte Zwangssituation vorlag und von der Frau als solche empfunden wurde ${ }^{23}$. Diese Formulierung wurde wiederholt bestätigt ${ }^{24}$. So kann durch eine vordergründig "gerecht « und angemessen erscheinende Einzelfallwürdigung ein Vorliegen von Gewalt nahezu beliebig angenommen oder ausgeschlossen werden ${ }^{25}$.

Die Anwendung eines engeren Gewaltbegriffes bei $\$ \$ 177,178 \mathrm{StGB}$ wird wie folgt begründet: Die $\$ \$ 177,178$ StGB enthalten höhere Anforderungen an die Drohungsmodalität als $\$ 240 \mathrm{StGB}$, daher müßten auch die Anforderungen an das Vorliegen von Gewalt höher $\operatorname{sein}^{26}$. Dafür spreche auch die Ausgestaltung der $\$ \int 177,178 \mathrm{StGB}$ als Verbrechen, während $\$ 240 \mathrm{StGB}$ ein Vergehen ist ${ }^{27}$. Schließlich gebe es in $\$ \$ \mathrm{I} 77$, $178 \mathrm{StGB}$ kein Korrektiv wie in $\$ 240$ II StGB, der Gewaltbegriff müsse diese Aussonderungsfunktion übernehmen ${ }^{28}$.

Diese Argumentation wird der speziellen Vergewaltigungssituation nicht gerecht. Der Gewaltbegriff in $\mathbb{\$} \$ 177,178 \mathrm{StGB}$ muß vielmehr auch unter Berücksichtigung der Opferperspektive ausgelegt werden ${ }^{29}$. Begründet in ihrer geschlechtsspezifischen Erziehung sind Frauen häufig auf eine physische Selbstverteidigung nicht vorbereitet. Sie geben in der Regel eine körperliche Gegenwehr schnell auf, wenn sie merken, daß damit die Brutalität des Übergriffes vergrößert wird ${ }^{3 \circ}$. Hinzu kommt, daß Vergewaltigungstäter Gewaltmittel oft in eskalierender Weise von psychischen Formen bis zu massivster Körpereinwirkung abhängig vom Widerstand des Opfers einsetzen $^{31}$.

Die Anwendung eines engen Gewaltbegriffes führt dazu, daß gerade in den Konstellationen keine Gewalt angenommen wird, in denen es zu keinem Zeitpunkt zu physischer Gewaltanwendung gekommen ist, die Frau aber gleichwohl ihre Lage als aussichtslos eingestuft hat ${ }^{32}$. Um eine vollendete Vergewaltigung zu beweisen, ist sie praktisch gezwungen, körperlichen Widerstand zu leisten, und damit ihr Verletzungsrisiko zu erhöhen. Die geleistete Gegenwehr wird an den sichtbaren Verletzungen gemessen 33 .

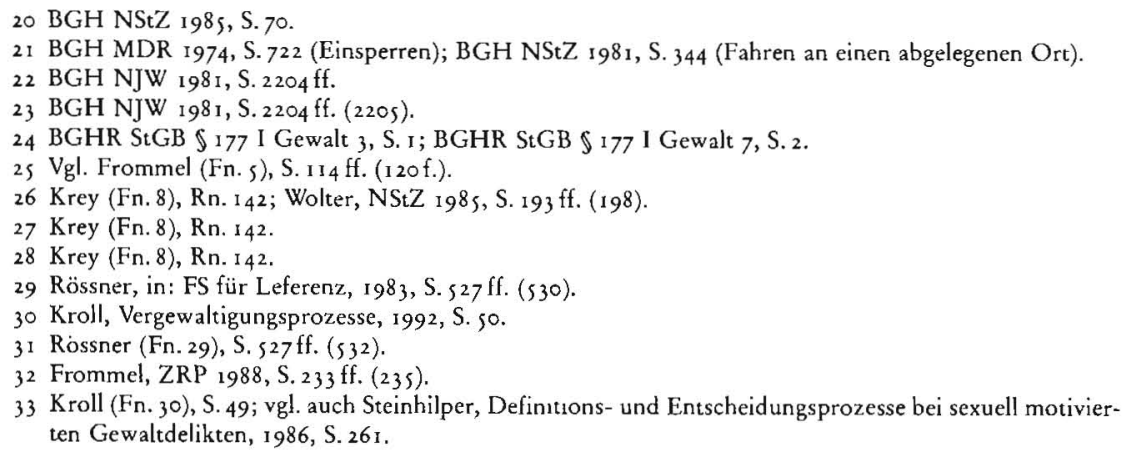


Die Kriterien der körperlichen Kraftentfaltung und der Körperlichkeit der Zwangseinwirkung beim Opfer sind zur Erfassung der spezifischen Vergewaltigungssituation nicht geeignet. Die fehlende klare Definition des Gewaltbegriffes in $\$ \$ 177,178$ StGB führt zu Rechtsunsicherheit, ein Prozeß bedeutet ein hohes Risiko der Nichtverurteilung, der in keinem Verhältnis zur erneuten hohen psychischen Belastung des Opfers steht.

\section{Final-funktionaler Zusammenhang zwischen Gewalt oder Drobung und sexueller Handlung/Beischlaf}

Als ungeschriebenes Tatbestandsmerkmal setzen die $\$ \$$ I 77 I, I78 I StGB eine finale Verknüpfung zwischen dem Einsatz des Nötigungsmittels und dem Beischlaf oder der sexuellen Handlung voraus ${ }^{34}$. Die Gewalt muß das Mittel zur Überwindung des Widerstandes $\operatorname{sein}^{35}$.

Auch dieses Erfordernis trägt dazu bei, eine Verurteilung wegen Vergewaltigung oder sexueller Nötigung unwahrscheinlicher zu machen. So urteilte der BGH in einem Fall, in dem der Angeklagte seine Tochter in einer Vielzahl von Fällen über zehn Jahre mißhandelt hatte, »um sie unter Ausnutzung der so zuvor geschaffenen Gewaltsituation zu sexuellen Handlungen und ... zum Geschlechtsverkehr zu zwingen«: Erforderlich für einen finalen Zusammenhang sei »die Feststellung von Tatsachen, aus denen sich ergibt, daß der Angeklagte gegen sein Opfer in unmittelbarem Zusammenhang mit sexuellen Handlungen Gewalt anwendete oder es bedrohte und daß das Opfer in anderen Fällen unter dem Eindruck der früheren Gewalt oder Drohung von einer Gegenwehr absah « ${ }^{36}$. Trotz einer »Aura der Angst «, die der Angeklagte um sich verbreitete, sah der BGH hier diese Tatsachen nicht hinreichend festgestellt.

Die subjektive Entsprechung dieses Tatbestandsmerkmals bietet ebenfalls Möglichkeiten, das Vorliegen einer Vergewaltigung oder sexuellen Nötigung abzulehnen. Der BGH stellte wiederholt ein Fehlen der Vorstellung vom Kausalzusammenhang zwischen Nötigung und Beischlaf fest, wenn der Täter Nötigungsmittel nur einsetzt, um das Opfer sexuell zu erregen und so zum freiwilligen Geschlechtsverkehr zu bewegen ${ }^{37}$.

Bei $\$ 178$ I StGB schließlich führt das Erfordernis des Finalzusammenhangs dazu, daß bei überraschend vorgenommenen sexuellen Handlungen eine sexuelle Nötigung i.S.d. $\$ 178$ I StGB nicht vorliegt: Der Tatbestand des $\$$ I78 I StGB setzt voraus, daß der Täter mittels Gewalt den Willen des Opfers zu beeinflussen oder seine Betätigung zu verhindern such $\mathrm{t}^{38}$. Es muß somit ein entgegenstehender Wille gebildet worden sein. Hieran fehlt es jedoch, »wenn der Täter die sexuelle Handlung so überraschend vornimmt, daß der Angegriffene einen Abwehrwillen nicht bilden konnte «39. Dem Täter gelinge der sexuelle Kontakt bereits durch die Überraschung der Opfer, nicht aufgrund der eingesetzten Gewalt. Ein latent vorhandener Wille, von ungewollten sexuellen Kontakten verschont zu bleiben, wird zwar anerkannt, bleibt aber ohne Einfluß.

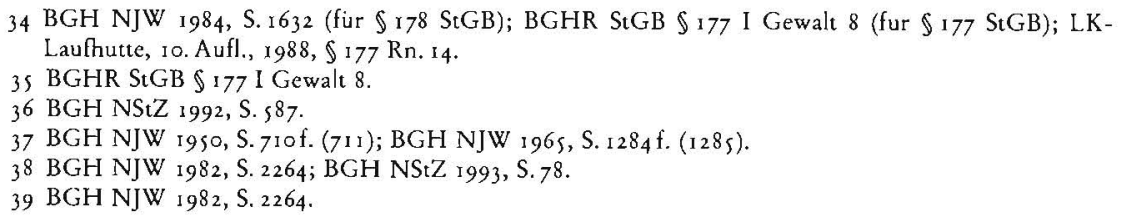


Die Drohungsalternative wird im Schrifttum häufig als Auffangtatbestand bei Nichtvorliegen von Gewalt i.S.d. $\$ \mathbb{S} 177$ I, 178 I StGB dargestellt ${ }^{40}$. Rechtsprechung und Literatur haben jedoch eine Erheblichkeitsschwelle entwickelt, bei deren Überschreiten erst eine tatbestandsmäßige Drohung vorliegen soll. Die Drohung muß eine Gefahr für Leib und Leben in Aussicht stellen. Dies erfordert »eine gewisse äußere Schwere des in Aussicht gestellten Angriffs auf die körperliche Unversehrtheit. Nicht jede Drohung mit einem Übel, das den Tatbestand der einfachen Körperverletzung erfüllt, ist schon eine Drohung mit Gefahr für Leib und Leben $\ldots \ll^{41}$. Die bloße Ankündigung von Schlägen reicht also nicht aus ${ }^{4^{2}}$.

Eine Begründung für die Einschränkung der Drohungsalternative auf eine erhebliche Leibesgefahr findet sich kaum. Der BGH weist an einer Stelle auf die "Zusammenstellung Leib oder Leben « hin $^{43}$. Es erscheint jedoch fragwürdig, aus der Tatsache, daß das höchste Rechtsgut vor Bedrohung geschützt wird, darauf zu schließen, daß der Schutz anderer, minder gewichtiger Rechtsgüter sich am Schutz dieses höchsten Rechtsguts orientieren muß ${ }^{44}$. Eine Restriktion der Drohung läßt sich aus dem Gesetz nicht zwingend ableiten. Sie kann einer Vergewaltigungssituation nicht gerecht werden, in der eine dem Täter ausgelieferte Frau sich allein schon aufgrund seiner überlegenen Körperkraft hilflos fühlt. Es entsteht eine Schutzlücke, da die Mehrzahl der Drohungen die Erheblichkeitsschwelle nicht überschreiten werden.

4. Sexuelle Handlungen und ibre Erheblichkeit (SS 178 I i. V.m. I84c Nr. I StGB)

$\$$ i 78 I StGB verlangt die Duldung einer sexuellen Handlung "an sich " oder die Vornahme einer solchen am Täter oder einem Dritten. Der Begriff der sexuellen Handlung bestimmt sich nach $\$ 184 \mathrm{c}$ Nr. 1 StGB. Danach sind nur solche Handlungen tatbestandsmäßig, die im Hinblick auf das geschützte Rechtsgut der sexuellen Selbstbestimmung von »einiger Erheblichkeit « sind. Entscheidend für das Vorliegen einer sexuellen Handlung ist ein »Bezug zum Geschlechtlichen «45, der nur für die Berührung der Geschlechtsteile und deren unmittelbare Umgebung gelten soll ${ }^{46}$. Ein "Dulden « der sexuellen Handlung "an sich" setzt nach der Rechtsprechung eine körperliche Berührung voraus, sie liegt nur vor, wenn der Täter »auf den Körper des Opfers einwirkt «47. Es genüge nicht, wenn lediglich die Kleidung des Opfers oder seine psychische Verfassung in Mitleidenschaft gezogen wird. Damit sieht der BGH ein Ejakulieren auf die Kleidung einer festgehaltenen Frau nicht als tatbestandsmäßige sexuelle Handlung an ${ }^{4}$.

Gleiches gilt für das gewaltsame Entfernen der Kleidung. Das »bloße Herunterreißen der Kleidungsstücke« stellt nach der Rechtsprechung "nicht den körperlichen Kontakt her, der für eine sexuelle Handlung i. S. d. $\$ \$$ I 78 , I $84 \mathrm{c} \mathrm{Nr}$. I StGB erforderlich ist «" 9 . Das Erfordernis der "Erheblichkeit « einer sexuellen Handlung in $\mathbb{I}_{\mathrm{r}} 84 \mathrm{c} \mathrm{Nr}$. I StGB ist im Gesetz nicht näher definiert. Es heißt lediglich, sie sei »im

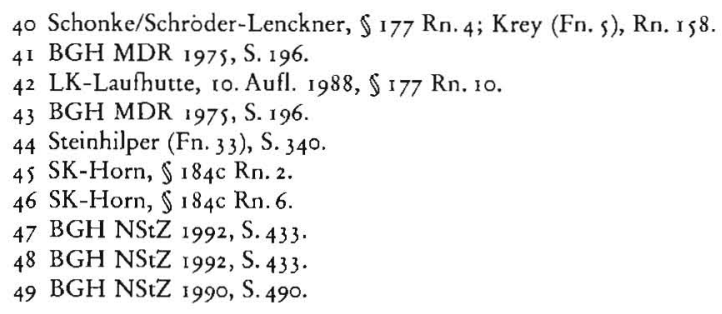


Hinblick auf das jeweilige Rechtsgut « zu bestimmen. Damit bleibt die Konkretisierung dem Ermessen des Gerichts überlassen. So werden hohe Anforderungen möglich, die zur Bagatellisierung sexueller Belästigungen führen.

Der Begriff »Beischlaf « in $\$ 177$ I StGB meint nur den Vaginalbeischlaf, nicht jedoch Oral- und Analverkehr. Diese sind nach $\$ 178 \mathrm{StGB}$ strafbar. Mit dem erzwungenen Beischlaf wird durch $₫ \mathbf{1 7 7}$ StGB ein besonders schwerer Eingriff in die körperliche Integrität des Opfers geahndet. Dieser ist beim erzwungenen Oral- oder Analverkehr mindestens ebenso gravierend, auch hier drohen traumatische Folgen ${ }^{\circ 0}$. Die Differenzierung ist aus Sicht der Opfer nicht begründbar.

Vergewaltigung und sexuelle Nötigung in der Ehe werden noch immer nicht als solche erfaßt. Sie können lediglich als Nötigung ( $\$ 240$ StGB), eventuell als Körperverletzung $(\mathbb{\$} 223 \mathrm{ff}$. StGB) bestraft werden. Die geltende Regelung wird folgendermaßen begründet: Fälle der Ehegattennotzucht hätten geringe Aufklärungschancen, der strafrechtliche Eingriff in den Intimbereich bringe gravierende Schwierigkeiten und Störungen des Ehelebens mit sich, eine Strafbarkeit gem. $\$ 240$ StGB sei ausreichend. Zudem gehörten geschlechtliche Bezichungen zum "Wesen der Ehe", die in $\llbracket 177,178 \mathrm{StGB}$ vorgesehene hohe Mindeststrafe könne sich in vielen Fällen als unangemessen hoch erweisen ${ }^{51}$.

Dem steht jedoch entgegen, daß die Beweisprobleme bei der Feststellung einer Nö-

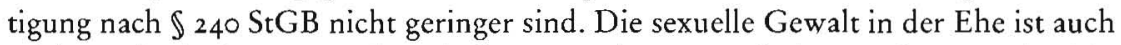
zahlenmäßig bedeutungsvolls ${ }^{\text {2 }}$ Ehefrauen sind vor spezifisch sexueller Gewalt nicht geschützt, der Nötigungstatbestand erfaßt den Angriff auf ihre sexuelle Freiheit nicht hinreichend.

\section{Fragwürdige Vorsatzkonstruktionen und "geschlechtsspezifische Situationsverkennung"}

Die Verwirklichung eines Straftatbestandes setzt Vorsatz des Täters voraus. Er muß den Willen zur Verwirklichung des Tatbestandes in Kenntnis aller objektiven Tatumstände haben ${ }^{53}$. Für $\mathbb{S} 177$ und I78 StGB bedeutet dies, daß die Nötigungsmittel eingesetzt werden müssen in der Absicht, den Beischlaf/die sexuelle Handlung zu ermöglichen ${ }^{54}$. Im übrigen genügt Eventualvorsatz ${ }^{55}$. Der Vorsatz des Täters ist naturgemäß schwer festzustellen, zur Klärung ist das Gericht auf indizielle Schlüsse angewiesen, die sich auf das bekundete Verhalten des Angeklagten und der betroffenen Frau stützen, wenn keine weiteren Beweise vorliegen ${ }^{56}$. Bei Zweifeln muß der Grundsatz »in dubio pro reo « gelten, doch zeigt sich, daß diesen Zweifeln - auch auf Grund überkommener Klischeevorstellungen - übermäßig leicht nachgegeben wird. Als Einfallstor in dieser Hinsicht bietet sich unter anderem eine fehlende Vorstellung des Täters vom Kausalzusammenhang zwischen Nötigung und Beischlaf/sexueller Handlung an oder ein Fehlen des Bewußtseins, daß sein Verhalten yom Opfer als Drohung aufgefaßt werden wirds7.

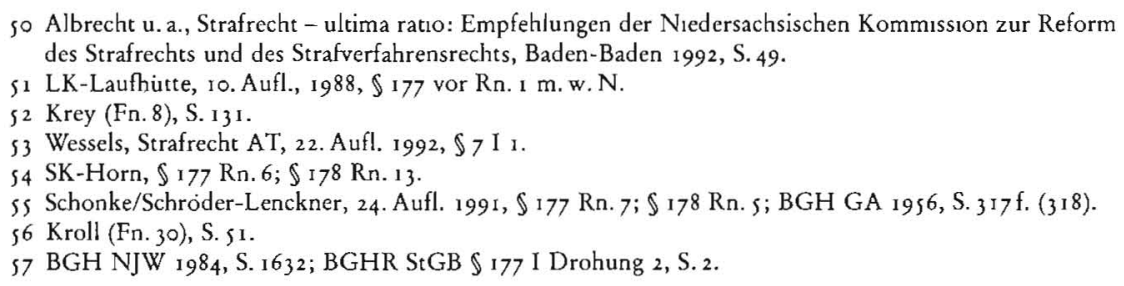


Insbesondere jedoch muß sich der Vorsatz des Täters darauf erstrecken, daß er » weiß oder billigend in Kauf nimmt «, durch das Nötigungsmittel einen »erwarteten oder begonnenen, ernsthaft gemeinten Widerstand « der Frau auszuschalten ${ }^{58}$. Er muß ihren Widerstand also erkennen und noch dazu ernstnehmen. Aus dieser von Rechtsprechung und Literatur ständig verwendeten Formel läßt sich schließen, daß es offenbar auch nicht »ernst gemeinten« Widerstand geben soll, daß eine Frau mit "Nein « eben häufig doch "Ja« meine. Dieses scheinbare Phänomen wird als "vis haud ingrata ", "nicht unwillkommene Gewalt« bezeichnet. Die Anerkennung der Möglichkeit, das Opfer habe sich nur "geziert", führt dazu, daß der Täter sich bei nur geringem Widerstand darauf berufen kann, er habe den Widerstand der Frau nicht für ernst gemeint gehalten und gedacht, sie sei mit seinem Vorhaben einverstanden gewesen (außer bei brutaler oder grober Gewalt). Dieser Irrtum über das Einverständnis wird von der Rechtsprechung und der überwiegenden Meinung im Schrifttum als Tatbestandsirrtum behandelt, der zum Vorsatzausschluß führt ${ }^{59}$. Hier sollen zwar "strenge Anforderungen " gestellt werden ${ }^{60}$, tatsächlich aber wird beim Vorliegen »nur « verbalen Widerstands dem Täter häufig eine "geschlechtsspezifische Situationsverkennung « zugute gehalten, in der er irrtümlich ein Einverständnis annehmen konnte und somit ohne Vorsatz handelte ${ }^{65}$.

Diese Konstruktion ist bei Anwendung der allgemeinen Regeln zur Vorsatzdogmatik zumindest fragwürdig. Handelt der Täter nämlich gegen einen erkennbaren Widerstand der Frau, stellt sich das Problem einer "geschlechtsspezifischen Situationsverkennung « im Grunde nicht. Der Täter müßte mit der von der Rechtsprechung verwendeten Möglichkeitstheorie zumindest einräumen, die konkrete Möglichkeit, der Widerstand sei ernstlich gewesen, in Betracht gezogen zu haben. Hat er dennoch sein Ziel weiterverfolgt, so hat er trotz Erkenntnis der konkreten Möglichkeit eines entgegenstehenden Willens sein Vorhaben in die Tat umgesetzt und damit vorsätzlich gehandelt ${ }^{62}$. Die Anerkennung der "vis haud ingrata " führt so auf der subjektiven Tatbestandsebene zu einer Ausweitung der Irrtumsmöglichkeiten. Im Prozeß wird eine klare Verweigerung einer sexuellen Handlung nicht als solche anerkannt, sondern nach Indizien gesucht, die den Täter an ein Einverständnis glauben lassen konnten ${ }^{63}$. Es ergibt sich also, daß ein auf Vergewaltigung oder sexuelle Nötigung gerichteter Vorsatz ein massiv körperlich vermitteltes ablehnendes Verhalten der Frau voraussetzt ${ }^{64}$.

\section{Strafböbe und minder schwerer Fall}

Der Mindeststrafrahmen für einen Regelfall der Vergewaltigung beträgt gem. $\$ 177$ I StGB zwei Jahre, der Regelstrafrahmen bei sexueller Nötigung ein bis zehn Jahre. Bei beiden Tatbeständen besteht die Möglichkeit, einen minder schweren Fall anzunehmen, dessen Strafrahmen gem. $\$ 177$ II StGB sechs Monate bis fünf Jahre, gem. $\mathbb{S}$ I 78 II StGB drei Monate bis fünf Jahre beträgt. Der große Anteil an Einstellungen

s8 BGH NStZ 1983, S. 71; vgl. LK-Laufhutte, 10. Aufl. 1988, \$ 177 Rn. 15; Schonke/Schroder-Lenckner, 24. Aufl. 1991, \177 Rn. 7; Lackner, StGB, 19. Aufl. 1991, \177 Rn.8

59 BGH GA 1970, S. 57; BGH NStZ 1983, S.71; LK-Laufhurte, 10. Aufl. 1988, \$177 Rn. I5; a. A. Maurach/Schroder/Maiwald, Strafrecht Besonderer Tell, Teilband 1, 7. Aufl. 1988, \$18 Rn. I 7 .

$60 \mathrm{BGH} \mathrm{GA}$ 1970, S. 57; BGH NStZ 1983, S. 71; BGH GA 1956, S. 317 f. (318) (danach sollte der bedingte Vorsatz nur dann fehlen, wenn sich der Tater uber die Einwilligung Gewißheit verschafft hat; diese Forderung wurde spater nicht mehr erhoben).

61 BGH NStZ 1982, S. 26; BGH NStZ 1983 , S. 71; BGHR StGB $\$ 177$ I Gewalt 8, S. 2.

62 Sick, ZStW 1991, S. 43 ff. (64).

$63 \mathrm{Vgl}$. Tondorf, StrV i 988 , S. 500 ff. ( $\left.\mathrm{SO}_{3}\right)$

64 Frommel, Zeitschrift fur Sexualforschung 1989, S. 132 ff. (137); vgl. auch Steınhilper (Fn. 33), S. 342. 
und Freisprüchen bei diesen Delikten zeigt, daß hohe Anforderungen an ihre Beweisbarkeit gestellt werden, bedingt durch die hohe Strafandrohung ${ }^{65}$. Zudem ist festzustellen, daß mit der Enge der Beziehung zwischen den Tatbeteiligten die durchschnittliche Strafhöhe sinkt, die Einstufung als »beweisschwierig" korreliert mit der Variable 'Täter-Opfer-Beziehung « ${ }^{66}$.

Um die hohe Strafdrohung zu vermeiden, entwickeln die Gerichte Umgehungsstrategien über den minder schweren Fall der $\$ 177,178 \mathrm{II} \mathrm{StGB}^{67}$. Zudem dürfen gem. $\$ 56$ II StGB nur Freiheitsstrafen zur Bewährung ausgesetzt werden, die zwei Jahre nicht übersteigen. So stellt die Einstufung eines Falles als "minder schwer " bei $\$ 177$ StGB die einzige Möglichkeit dar, eine Freiheitsstrafe zur Bewährung auszusetzen. Dies hat dazu geführt, daß der minder schwere Fall extensiv angewendet wird, faktisch kehrt sich das Rangverhältnis um, der minder schwere Fall wird zum Normalfall ${ }^{68}$. Im Prozeß wirkt sich diese Tatsache für die betroffene Frau belastend aus. Die Begründung des minder schweren Falles erfolgt regelmäßig in der Weise, daß dem Opfer eine mehr oder weniger große Mitschuld zugewiesen wird. ${ }^{69}$

Da das Gesetz keine Kriterien für das Vorliegen eines minder schweren Falles liefert, ist ihre Ausarbeitung Literatur und Rechtsprechung überlassen. Es können "grundsätzlich nur besondere, nicht alltägliche Umstände« ein Abweichen vom Regelstrafrahmen rechtfertigen. « ${ }^{70}$

Hierzu zählt vor allem eine schon vor der Tat bestehende Beziehung zwischen Täter und $\mathrm{Opfer}^{7}$. Durch die Einschätzung von Beziehungstaten als "minder schwer" werden diese bagatellisiert ${ }^{72}$. Entgegen der rechtlichen Einschätzung belegen Untersuchungen, daß auch sexuelle Gewalt im sozialen Nahraum einschneidende Folgen für die Opfer hat, da Beziehungsgewalt immer mit einem Vertrauensbruch einhergeht ${ }^{73}$. Von einem »minder schweren Fall« kann also keine Rede sein. Auch »tatförderndes, die Tatdurchführung erleichterndes und deshalb , beihilfenahes، Opferverhalten «74 gibt häufig Anlaß zur Annahme eines minder schweren Falles ${ }^{75}$. Hier wird dem Opfer die Initiative und Verantwortung für das Verhalten des Vergewaltigers zugewiesen, eine Frau hat "Gefahrensituationen« tunlichst zu vermeiden. Auch das Phänomen der "geschlechtsspezifischen Situationsverkennung « wird zur Strafmilderung herangezogen. Es gebe Situationen, in denen der Täter "Verführung und Vergewaltigung innerlich vermischt hat ${ }^{76}$. Dem scheint ebenfalls die Idee der "vis haud ingrata" zugrundezuliegen, es wird dem Täter nicht zugemutet, die von der Frau gesetzten Grenzen zu erkennen und zu akzeptieren. In diesem Zusammenhang taucht auch immer wieder der »sexuelle Notstand « auf ${ }^{77}$. Diese Vorstellung verkennt den Charakter der überwiegenden Zahl von Sexualverbrechen. Vergewaltigungsmo-

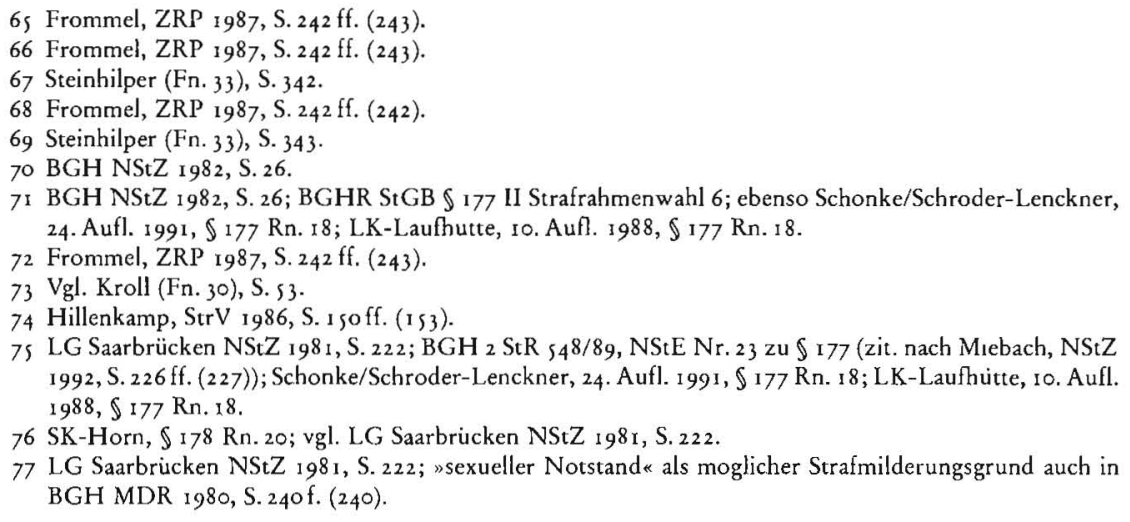


tiv ist in der Regel nicht die Befriedigung sexueller Triebe, sondern die Unterwerfung und Demütigung des Opfers ${ }^{78}$.

Diese zur Rechtfertigung eines minder schweren Falles herangezogenen Umstände, die ja nach der Formel der Rechtsprechung »vom Durchschnitt der gewöhnlichen Fälle « abweichen müssen, lassen erkennen, daß nach wie vor die in der Praxis verhältnismäßig seltene überfallartige Vergewaltigung für den Durchschnittsfall gehalten wird. In der weit überwiegenden Mehrheit der Fälle jedoch kannten Täter und Opfer sich, eine große Zahl von Vergewaltigungen findet in Wohnungen statt ${ }^{79}$. Vor allem die hohe Strafdrohung führt so zu selektiver Strafverfolgung. Dies wirkt dem Opferschutz entgegen ${ }^{80}$.

Was sich in der Rechtsprechung zeigt, spiegelt sich in der Reformdiskussion wieder.

\section{Die Reformdiskussion}

\section{Entwicklung und Verlauf}

Erste Phase 1973-1982:

Im Jahr 1973 wurde durch das 4. Strafrechtsreformgesetz festgestellt, daß die Sexualdelikte nicht mehr als Verbrechen gegen die Sittlichkeit (weibliche Geschlechtsehre) einzustufen sind, sondern Verbrechen gegen das sexuelle Selbstbestimmungsrecht der Frau sind. Damit wurde anerkannt, daß sexuelle Gewalt ein schwerer Verstoß gegen die Menschenwürde und die Freiheitsgarantien ist. Eine Veränderung der Gesetzestexte der $\$ \$ 177 \mathrm{ff}$. StGB wurde kaum vorgenommen und blieb folgenlos. Die klassische Definition des Gewaltbegriffs wurde bewußt nicht verändert.

\section{Zweite Phase 1983-1990}

Im Jahr 1983 machte die damalige Hamburger Justizsenatorin Eva Leithäuser den Vorstoß, die Vergewaltigung in der Ehe in den $\$ 177$ StGB explizit aufzunehmen. Seitdem werden Gesetzesentwürfe zu Reform der sexuellen Gewaltdelikte diskutiert. In einer ersten Phase sollte ausschließlich die Vergewaltigung in der Ehe pönalisiert werden. Erst später wurde auch die Situation der vergewaltigten Frau im Strafverfahren thematisiert. Erste Verbesserungen konnten dabei durch das Opferschutzgesetz von 1986 vorgenommen werden.

Ende der 8 oer Jahre lagen verschiedenste Entwürfe vor, deren einzige Übereinstimmung zunächst die Streichung des Merkmals "außerehelich"war. Ein Teil der Entwürfe strebte eine umfassende Reform an, d. h. der deliktspezifisch enge Gewaltbegriff sollte im Gesetz erweitert werden, die Mindeststrafe sollte gesenkt werden (von zwei Jahren auf ein Jahr) und der minderschwere Fall sollte abgeschafft werden. Zu diesen Entwürfen zählten der Entwurf der GRÜNEN, der des Juristinnenbundes und der des damaligen Bundesfamilienministeriums (BM JFFG). Im Gegensatz zu diesen sah ein anderer Entwurf des Bundesjustizministeriums mehr Spielräume für richterliche Einzelfallentscheidungen vor, neben der Senkung der Mindeststrafe sollte auch eine weitere Strafmilderung bei »minder schweren Fällen « möglich sein ${ }^{81}$.

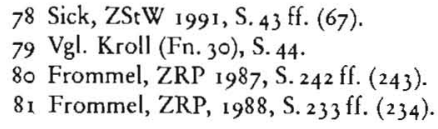


Unterschiede zwischen den genannten Entwürfen betreffen die Auffassung der sexuellen Nötigung als Vergehen oder Verbrechen ${ }^{82}$. In den Entwürfen finden sich auch unterschiedliche Vorschläge als Alternativen zum Strafrecht (im Sinne des Täter-Opfer-Ausgleichs). In Kombination dieser informellen Verfahren mit den strafrechtlichen Regelungen gab es folgende Vorschläge ${ }^{8_{3}}$ :

- Informelle Absprachen im Rahmen des formellen Programms des Prozeßrechts. Beispiel: Bei einer positiven Prognose die Strafaussetzung zur Bewährung. Einwand: nur akzeptabel bei Vergehen.

- Strafantragsrecht der Ehefrau. Einwand: Sollte wie bisher nur bei Vergehen möglich sein, da Verbrechen Offizialdelikte sind.

- Absehen von Strafe, wenn dies im Interesse der Aufrechterhaltung der Bindungen liegt. Einwand: Umsetzung wird unkontrolliert der Praxis überlassen. Sexuelle Gewalt in Beziehungen ist kein punktuelles Ereignis. Dieser Vorschlag wird der destruktiven Dynamik von Gewaltbeziehungen nicht gerecht, sondern verstärkt diese eher.

- Strafvorbehalt bei einer Einigung zwischen Täter und Opfer. Das Gericht entscheidet über Prognose im Falle von Therapie oder Einigung. Einwand: s.o.

\section{Aktueller Stand der Reformdiskussion}

Die Gesetzesentwürfe:

Dem Deutschen Bundestag liegen in dieser i 2. Wahlperiode die folgenden drei Gesetzesentwürfe zur Änderung des Sexualstrafrechtes zur Beschlußfassung vor ${ }^{8_{4}}$ :

I. Entwurf der SPD-Fraktion vom i 2. I 2. I 99 I.

2. Entwurf des Bundesrates unter Federführung des Bundesministers der Justiz vom 26. 2. 92 .

3. Entwurf der Abgeordneten Christina Schenk und der Gruppe Bündnis 90/Die Grünen vom 24.9.92.

Der Entwurf des Deutschen Juristinnenbundes ist hier aus Platz- und Aktualitätsgründen nicht aufgeführt. Er hat jedoch einen maßgeblichen Beitrag zum Vorangehen der Reformdiskussion geleistet. Der Entwurf der Niedersächsischen Kommission zur Reform soll hier kurz zusammengefaßt wiedergegeben werden ${ }^{85}$. Dieser Entwurf integriert die grundlegende Neudefinition des Gewaltbegriffs: ein einheitlicher enger Gewaltbegriff soll für alle Delikte gelten.

Die Reformvorschläge der Niedersächsischen Kommission.

Die Vorschläge der Niedersächsischen Kommission zur Reform des Strafrechts fordern, das geltende Strafrecht am Grundrecht auf sexuelle Selbstbestimmung zu messen, und schlagen dazu vor, dies durch das Kernstrafrecht zu gewährleisten. Strafrecht hat einzugreifen, wenn "Zwang, Ausbeutung oder »Mißbrauch« vorliegen. Dabei sollte die Stellung des Opfers betont werden. Die Legaldefinition von Gewalt soll in $\$ i i Abs. I Nr. ro StGB aufgenommen werden: Gewalt ist "Zwang, der mittels Einwirkung auf den Körper eines anderen Menschen oder mittels einer Freiheitsberaubung $(\$ 329 \mathrm{StGB})$ ausgeübt wird «. Den Vorschlägen wird die Ergän-

\footnotetext{
82 Frommel, ZRP, 1988, S. 233 ff. (238).

83 Frommel, ZRP 1987 , S. 242 ff. (242)

84 Deutscher Bundestag, 12. Wahlperiode: Drucksache I 2/1818 vom I 2. 1 2. 9 I: Gesetzentwurf der Fraktion der SPD. Drucksache 12/2167 vom 26. 2.92: Gesetzentwurf des Bundesrates. Drucksache 12/3303 vom 24.9.91: Gesetzentwurf der Abgeordneten Christına Schenk und der Gruppe BÜNDNIS 90/DIE GRÜNEN.

85 Albrecht u. a. (Fn. 50), S. 44 ff.
} 
zung einer Legaldefinition in $\$$ I $84 \mathrm{C}$ StGB vorangestellt. Danach ist das Merkmal „Beischlaf « durch das Merkmal "Geschlechtsverkehr in den jeweiligen Paragraphen zu ersetzen und erzwungener Anal- und Oralverkehr sind dem erzwungenen Vaginalverkehr gleichzustellen. Die Vorschläge im einzelnen:

Vergewaltigung ( $\int 177$ St $\left.G B\right)$ :

- Im Zuge der Gleichstellung der Geschlechter sollte der Opferkreis erweitert werden, somit wird auch die Strafverfolgung der Vergewaltigung von Männern durch Männer möglich.

- Das Tatbestandsmerkmal »außerehelich « sollte gestrichen werden: Ein relevanter Teil von Ehefrauen wurde schon einmal vom Ehemann zum Geschlechtsverkehr gezwungen.

- Zur Definition der Nötigungsmittel schlägt die Kommission den oben genannten von ihr entwickelten allgemeinen Gewaltbegriff zur Anwendung vor. So wird das Erfordernis einer körperlichen Einwirkung klargestellt und Freiheitsberaubung als Nötigungsmittel anerkannt.

- Der Strafrahmen sollte eine Senkung der Mindeststrafe von zwei auf ein Jahr Freiheitsstrafe vorsehen bei gleichzeitiger Streichung des minderschweren Falles ( $\$$ I 77 Abs. 2 StGB). Ein anderer Teil der Kommission plädierte für die Beibehaltung des geltenden Rechts aufgrund der Verwerflichkeit des Verbrechens.

- Es wird vorgeschlagen dem Opfer ein Widerspruchsrecht einzuräumen: »Die Tat ist nicht zu verfolgen, wenn die verletzte Person widerspricht «. Ein anderer Teil der Kommission sah keinen Anlaß für eine Sonderregelung: Es handelt sich um ein Offizialdelikt, Widerspruchsmöglichkeiten eröffnen die Möglichkeit, Zwang und Druck auf das Opfer auszuüben und könnten wohlhabende Täter privilegieren.

- Neuformulierung $\$ 177$ :

(I) Wer eine andere Person mit Gewalt ( $\$ 1$ I Abs. I Nr. Io StGB) oder durch Drohung mit Gewalt oder mit gegenwärtiger Gefahr für Leib und Leben zum Geschlechtsverkehr ( $\$$ I $84 \mathrm{c}$ Nr. I StGB) mit sich oder einem Dritten nötigt, wird mit Freiheitsstrafe nicht unter einem Jahr bestraft.

(2) Wird durch die Tat leichtfertig der Tod des Opfers verursacht, so ist die Freiheitsstrafe nicht unter fünf Jahren.

(3) Die Tat ist nicht zu verfolgen, wenn die verletzte Person widerspricht.

Sexuelle Nötigung ( 1778 StGB):

- Das Tatbestandsmerkmal »außerehelich « sollte gestrichen werden.

- Als Nötigungsmittel sollte hier auch die Drohung mit Gewalt als Tathandlung betrachtet werden.

- Sexuelle Nötigung sollte als Vergehen konzipiert werden: Der Strafrahmen sollte keine Mindeststrafe angeben und nur die Höchststrafe von zehn Jahren beibehalten.

- Der Versuch einer sexuellen Nötigung sollte strafbar sein.

- Neuformulierung $\$ 178$ StGB:

(I) Wer eine andere Person mit Gewalt oder durch Drohung mit Gewalt oder Gefahr für Leib und Leben nötigt, sexuelle Handlungen des Täters oder einer dritten Person an sich zu dulden oder an dem Täter oder einer dritten Person vorzunehmen, wird mit Freiheitsstrafe bis zu zehn Jahren bestraft.

(2) Der Versuch ist strafbar.

(3) Wird durch die Tat leichtfertig der Tod des Opfers verursacht, so ist die Freiheitsstrafe nicht unter fünf Jahren.

(3) Die Tat ist nicht zu verfolgen, wenn die verletzte Person widerspricht.

Der Vollständigkeit halber sei noch erwähnt, daß die Reformkommission Vorschläge zu den $\$ \$ 179$ und 174 StGB, die jedoch nicht weiter ausformuliert wurden, und zur 
Entkriminalisierung der »Unzuchtsdelikte" gibt. Bei letzteren handelt es sich um sehr grundlegende Veränderungen, bei denen die Beschränkung auf das Kernstrafrecht deutlich wird.

\section{Bewertung der Reformdiskussion}

Aus diesem Überblick zur Reformdiskussion wird deutlich, daß eine "Reform « nicht in Sicht ist: Zwar soll die Vergewaltigung in der Ehe unter Strafe gestellt werden, durch die Hintertür schleicht sich jedoch in Form des Absehens und der Milderung von Strafe die alte Ungleichbehandlung von verheirateten und nicht-verheirateten Tätern durch den Gesetzgeber wieder ein. Auch die Neudefinition des $\$ 178 \mathrm{StGB}$ als Vergehensstraftatbestand wird ebenso versäumt wie die Möglichkeit, den sogenannten »kleinen « Sexualdelikten einen Platz im Strafrecht zukommen zu lassen. Diese Veränderung könnte es jedoch durch ihre Differenzierung ermöglichen, eine Analogie zur Abstufung der Schwere von Sexualstraftaten herzustellen, wie sie in der Praxis vorzufinden ist. Somit wäre eine Beschlußfassung für ein neues Gesetz »eine Reform, die keine ist«.

Der Entwurf mit der größten Wahrscheinlichkeit einer politischen Umsetzung, der Bundesratsentwurf, umgeht die entscheidenden Fragen des Nötigungsmittels und der Strafzumessung.

Zur Zeit bestätigt sich hinsichtlich des Gewaltbegriffs Monika Frommels Einschätzung des Scheiterns der Reform an politischen Widerständen ${ }^{86}$ : Der Entwurf zur Vereinheitlichung des strafrechtlichen Gewaltbegriffs und der Reform des $\$ 24^{\circ}$ StGB (Gesetzesantrag des Landes Niedersachsen) wurde im Rechtsausschuß am I0.3.93 gegen die Stimmen Bayerns und Sachsens vertagt, da sich z. B. die Frage "unerwünschter Strafbarkeitslücken« als ungeklärt erwies. Der Vertreter Bayerns kündigt für weitere Beratungen eine Ablehnung des Entwurfs an, da in dem Entwurf der "Begriff der `Gewalt< unvertretbar weit eingeschränkt" sei ${ }^{87}$. Ähnlich erging es dem Gesetzesentwurf in den Ausschüssen für Familie und Jugend sowie für innere Angelegenheiten ${ }^{88}$. Jetzt kann abgewartet werden, ob auch Frommels abschließende Einschätzung zutrifft, daß »die Mehrheit in Bundestag und Bundesrat ... daraufhin jede Reform blockieren « wird. Im übrigen wäre es auch bei Verwendung dieses klarstellenden engen Gewaltbegriffs notwendig, adäquate Mißbrauchstatbestände zu schaffen, die die nicht erfaßten Fälle der Einschüchterung und Überrumpelung körperlich unterlegener Opfer unter Strafe stellen ${ }^{89}$.

\section{Schlußbetrachtung}

Die unterschiedliche Interpretation von Gewalt in den verschiedenen gesellschaftlichen Gruppen wurde gezeigt: Dabei zeichnet sich eine Dynamik des Auseinanderdriftens von allgemeinen politischem Diskurs und fachspezifischem Diskurs ab. Es besteht eine unterschiedliche Wahrnehmung von Gewalt und Sexualität bei Frauen und Männern. Ein markanter Beleg ist die oben dargestellte Konstruktion der »vis haud ingrata " und die damit zusammenhängende höchstrichterliche Rechtsprechung. Darüber hinaus ist eine weitere Differenz zwischen mit Sexualdelikten

86 Frommel, Monika: Vorüberlegungen zu einer umfassenden Reform des Sexualstrafrechts. Vortrag auf dem Alternativen Juristentag, 1992.

87 Auszug aus dem Protokoll der 659. Sitzung des BR-Ausschusses fur Recht am 10.3.93.

88 Auszug aus dem Protokoll der 21. Sitzung des BR-Ausschusses fúr FJ am 10.3.93 und Auszug aus dem Protokoll der 656. Sitzung des BR-Ausschusses fur Innere Angelegenheiten am 11.3.93. 89 Frommel (FN 86), S. 16. 
professionell Befaßten und von Gewalt Betroffenen deutlich geworden. Anscheinend entwickeln sich Gesetzgebung, Gerichte, Staatsanwaltschaft, Polizei, Beratungsstellen und Betroffene im Umgang mit Gewalt auseinander: Es bestehen unterschiedliche Interpretations- und Verhaltensmuster. Bedeutsam ist hier für die Zukunft ein Austausch aller Beteiligten, um einen Bewußtseinswandel gerade auch in der höchstrichterlichen Rechtsprechung zu erreichen. Ohne die Entwicklung eines vorurteilsfreien Umgangs mit Sexualdelikten können keine grundlegenden Veränderungen erzielt werden. Punktuelle Reformen würden lediglich zu neuen Umgehungsstrategien der Gerichte führen.

Der Gewaltbegriff muß im Hinblick auf das Rechtsgut der sexuellen Selbstbestimmung neu definiert werden. Bis heute wird von den Frauen "geschlechtsrollenkonformes Verhalten erwartet, z. B. bestimmte Situationen zu vermeiden. Diese vorrechtliche(n) Stereotypien schränken die Umsetzung des Strafrechts auf eine dem Opfer nicht dienliche Weise ein. Die Entkriminalisierung im Falle von Beziehungstaten macht private Gewalt zur Privatsache und führt zu fehlendem Schutz der sexuellen Selbstbestimmung. Ein öffentliches Interesse liegt jedoch vor und die Konsequenz sollte Strafverfolgung sein, da grundlegende individuelle Rechte verletzt werden.

\section{Lorenz Petersen}

\section{Zwischen Mythos und Misere: Die amerikanische Umweltbehörde EPA*}

\section{Einfübrung}

Umweltpolitik ist in die Jahre gekommen und mit ihr ihre staatliche Organisationsweise. Parallel zur institutionellen Etablierung des Umweltschutzes seit einer Aufbruchphase Anfang der siebziger Jahre haben sich Vorstellungen von der Beherrschbarkeit des Umweltproblems angesichts immer neuer krisenhafter Zuspitzungen und schleichender Gefährdungen stark relativiert. Ein prominentes Beispiel staatlicher Umweltschutzbemühungen ist die amerikanische Umweltbehörde EPA (Environmental Protection Agency), die seit ihrer Gründung 1970 eine ausgesprochen bewegte, wechselvolle Geschichte erlebte. Prominent heißt nicht auch gleich beliebt. Denn die Position der Behörde zwischen Kongreß und Weißem Haus, gerichtlicher Kontrolle und dem über Lobbygruppen kanalisierten Teil der öffentlichen Meinung ist ausgesprochen diffizil. Nicht selten resultierte aus dieser Konstellation eine phasenweise Lähmung der EPA-Aktivitäten in wichtigen umweltpolitischen Fragen.

Im internationalen Vergleich galten die »policies « US-amerikanischer Umweltpolitik vor allem in den siebziger Jahren als innovativ, und die EPA wurde und wird besonders von Ökonomen im Zusammenhang mit der Anwendung von Zertifikatslösungen in der Luftreinhaltepolitik gern zitiert. Um so mehr wurde die Haltung der Bush-Administration auf der UN-Umweltkonferenz in Rio de Janeiro zum Ausdruck für den geringen Stellenwert des Umweltschutzes gegenüber nationalen

* Gedankt sei an dieser Stelle dem Zentrum für Nordamerikaforschung in Frankfurt/Main und der Stiftung Volkswagenwerk fur die Gewahrung eınes insgesamt sechsmonatıgen Internship-Stipendiums, durch das der Autor im Winter/Fruhjahr 1990/91 Gelegenheit hatte, das "Innenleben « der Environmental Protection Agency kennenzulernen. 